\title{
Médiévales
}

Langues, Textes, Histoire

65 | automne 2013

Le couple dans le monde franc

\section{Un homme, une femme et des enfants : autour du couple dans quelques images du haut Moyen Âge}

Man, Woman and Children : the Couple in a Few Images of the Early Middle Ages

Dominique Alibert

\section{OpenEdition}

1 Journals

Édition électronique

URL : https://journals.openedition.org/medievales/7098

DOI : 10.4000/medievales.7098

ISSN : 1777-5892

Éditeur

Presses universitaires de Vincennes

Édition imprimée

Date de publication : 1 décembre 2013

Pagination : 65-75

ISBN : 978-2-84292-396-9

ISSN : 0751-2708

Référence électronique

Dominique Alibert, « Un homme, une femme et des enfants: autour du couple dans quelques images du haut Moyen Âge », Médiévales [En ligne], 65 | automne 2013, mis en ligne le 20 janvier 2014, consulté le 23 avril 2022. URL : http://journals.openedition.org/medievales/7098 ; DOI : https:// doi.org/10.4000/medievales.7098

Tous droits réservés 
Médiévales 65, automne 2013,p. 65-76

Dominique ALIBERT

\section{UN HOMME, UNE FEMME ET DES ENFANTS : AUTOUR DU COUPLE DANS QUELQUES IMAGES DU HAUT MOYEN ÂGE}

Dans la bibliographie classique, notamment depuis un article de Pierre Toubert qui fit date ${ }^{1}$, l'époque carolingienne est vue comme un temps de réflexion sur la place que l'Église entend donner au mariage. Paradoxalement ${ }^{2}$, les documents iconographiques qui évoquent le couple, ou même le mariage, sont plus rares: ils n'excèdent pas la quinzaine et surtout, ce qui complique encore l'analyse, ils proviennent pour l'essentiel de trois manuscrits ${ }^{3}$.

Ce corpus a été constitué après la consultation de l'essentiel des grands manuscrits enluminés carolingiens et des principales reliures sculptées qui nous sont parvenus. Ont été exclues les images d'Adam et Ève avant et après la Chute, car ces dernières sont relativement stéréotypées et le peintre dispose rarement d'une grande latitude ${ }^{4}$.

1. P. TOUBERT, «La théorie du mariage chez les moralistes carolingiens », dans Il matrimonio nella società altomedievale, Settimane, 24 (1977), p. 235-285, repris dans ID., L'Europe dans sa première croissance. De Charlemagne à l'An Mil, Paris, 2004, p. 283-309.

2. Ou habituellement? Souvent les images et les textes ne parlent pas forcément des mêmes réalités ou n'utilisent pas les mêmes temporalités.

3. J'ai volontairement laissé de côté les images ottoniennes car elles représentent un tout autre ensemble, que pour l'heure je ne maîtrise pas encore tout à fait, et il n'était pas intellectuellement satisfaisant de présenter deux ensembles sans comprendre ce qui pouvait les unir ou les séparer.

4. On peut citer, à titre d'exemple, le folio $10 \mathrm{v}^{\circ}$ de la Première Bible de Charles le Chauve, Paris, BnF, lat. 1, http://visualiseur.bnf.fr/CadresFenetre?O=COMP-1\&M=imageseule. Si on la compare avec deux images contemporaines ou postérieures de quelques décennies, celle de la Bible de Moutier-Grandval et celle de la Bible de Saint-Paul-hors-les-Murs, on s'aperçoit qu'elle est relativement stéréotypée. Il est dès lors délicat de tenter de tirer quelque information de ce type d'images. Bible de Moutier-Grandval, Londres, British Museum (Add. Ms. 10.546), fo 7v $\mathrm{v}^{\circ}$. Bible de Saint-Paul-hors-les-Murs, Rome, Saint-Paul-hors-les-Murs, $\mathrm{f}^{\circ} 18 \mathrm{v}^{\circ}$. 
Nous avons, pour des raisons similaires, éliminé les représentations de Marie et Joseph. Citons pour mémoire l'un des derniers folios du Psautier d'Utrecht (89 verso) qui illustre le Cantique de Siméon ${ }^{5}$. Ce texte, également connu par son premier verset nunc dimittis, est inséré dans le récit que donne Luc de la présentation au Temple ${ }^{6}$. Le dessinateur a choisi, selon une tradition iconographique promise à une longue descendance, de mettre en avant Marie tendant l'Enfant au vieillard Syméon, l'un et l'autre sur les marches du Temple, tandis que Joseph se tient en retrait, avec son panier dans lequel il a pris soin de placer, selon le récit évangélique ${ }^{7}$, des colombes.

Le Psautier d'Utrecht ${ }^{8}$ évoqué plus haut fournira, avec le Psautier de Stuttgart ${ }^{9}$, plus de $80 \%$ des images que nous analyserons. La reliure du Psautier de Charles le Chauve ${ }^{10}$ et la Bible de Saint-Paul-hors-les-Murs ${ }^{11}$ compléteront l'ensemble. Le premier des deux psautiers cités, conservé aujourd'hui à la Bibliothèque universitaire d'Utrecht, est issu du scriptorium rémois et date très probablement des années $823^{12}$. Le second, que possède maintenant la Bibliothèque du Land de Bade-Wurtemberg à Stuttgart, a probablement été réalisé à Saint-Germain-des-Prés au tournant des deux premières décennies du $\mathrm{IX}^{\mathrm{e}}$ siècle $^{13}$.

Malgré sa faiblesse numérique, le corpus que nous avons réuni nous permettra de préciser les fonctions du couple dans l'iconographie carolingienne. Nous tenterons de les définir en analysant deux couples hors du commun, David et Bethsabée, Charles le Chauve et Richilde, en passant par la représentation des

5. http://objects.library.uu.nl/reader/index.php?obj=1874-99999\&lan=en\#page//psalter/ FOL_089V.jpg/mode/1up.

6. Lc, 2, 29-32: «Nunc dimittis servum tuum Domine, Secundum verbum tuum in pace: Quia viderunt occuli mei salutare tuum, Quod parasti ante faciem omnium populorum: Lumen ad revelationem gentium, et gloriam plebis tuae Israel» (Maintenant, Maître, c'est en paix comme tu l'as dit que tu renvoies ton serviteur. Car mes yeux ont vu ton salut que tu as préparé face à tous les peuples : lumière pour la révélation aux païens et gloire d'Israël ton peuple.) Les citations bibliques sont données d'après une Vulgate clémentine. Les traductions sont celles de la Bible de Jérusalem, utilisée pour des raisons éditoriales tout en étant conscient que cette dernière présente souvent un écart avec la Vulgate.

7. Lc, 2, 24: «Et ut darent hostiam secundum quod dictum est in lege domini par turturum, aut duos pullos collumbarum.» (Et pour offrir en sacrifice, suivant ce qui est dit dans la loi du Seigneur, un couple de tourterelles ou deux petits pigeons.)

8. Utrecht, Rijksuniversiteit te Utrecht. Bibliotheek, ms. 32.

9. Stuttgart, Württembergische Landesbibliothek, Cod. bibl. fol. 23.

10. Paris, BnF, lat. 1152 .

11. Rome, Saint-Paul-hors-les-Murs.

12. Pour la datation des manuscrits, J.-P. CAILlET, L'Art carolingien, Paris, 2005, p. 179-182. Sur certains points, on nous permettra de renvoyer à D. ALIBERT, Les Carolingiens et leurs images. Iconographie et idéologie, Thèse dactylographiée, Paris-IV, 1994, p. 313-315.

13. Ibid., p. 296-297. 
couples plus ordinaires. Précisons que la nature des documents envisagés fait que ce sont les textes qui accompagnent les images qui permettent de définir les hommes et les femmes représentés comme constituant un couple.

\section{Les amants, le Hittite et le prophète}

Il existe trois images d'un couple adultère. Ou, pour être plus précis, une première image et une seconde qui est constituée des deux exemplaires d'une même matrice, puisque la seconde image s'inspire visiblement de la première. Il s'agit d'une enluminure du Psautier d'Utrecht qui a été reprise par l'ivoirier qui a orné la reliure du Psautier de Charles le Chauve. Néanmoins les écarts entre la réplique et son modèle font sens.

La première est l'illustration du verset 1 du très célèbre psaume 50 , passé à la liturgie et la musique sous le titre de Miserere, qu'il tire de son deuxième verset. Psaume pénitentiel par excellence, c'est son premier verset, narratif, qui va nous retenir: "Quand le prophète Nathan alla chez lui, après que David fut allé chez Bethsabée ${ }^{14}$.» Il s'agit là d'une allusion à l'épisode narré par le Second livre de Samuel, dans ses chapitres 11 et 12: l'histoire de David et Bethsabée. L'irritation de Yahvé, provoquée par l'adultère puis le meurtre, conduit Nathan à chapitrer David, coupable d'avoir méprisé la parole divine ${ }^{15}$.

Dans le Psautier d'Utrecht ${ }^{16}$, Bethsabée se tient à l'entrée latérale d'un bâtiment qui peut être assimilé, sans forcer le document, à un palais royal. Le rideau qu'elle écarte a probablement une fonction séparatrice entre l'espace public et l'espace privé. David, vêtu en laïque, manteau court retenu par une fibule à l'épaule, ébauche un geste d'étonnement qui établit aussi un lien entre Bethsabée, située derrière lui, et Nathan. Il porte sa couronne. Nathan, à la droite de la scène, semble dans une sombre colère alors qu'il fait un geste pour semondre le roi. Le reste de l'image est constitué par l'illustration de la parabole de l'homme pauvre et de l'homme riche. En léger contrebas du palais se trouve un cadavre, bien évidemment celui d'Urie.

Il est intéressant de comparer les deux versions du même épisode à quarante ans d'écart ${ }^{17}$.

14. Ps. 50: «cum venit ad eum Nathan propheta quando intravit ad Bethsabee».

15. 2 Sam 12,9. David s'éprend de Bethsabée, femme de l'un de ses généraux, Urie le Hittite, et fait tuer ce dernier. 2 Sam 11.

16. Psautier d'Utrecht, Utrecht, Rijksuniversiteit te Utrecht. Bibliotheek, ms. 32, fo $29 \mathrm{r}^{\circ}$. http://objects.library.uu.nl/reader/index.php?obj=1874-284427\&lan=en\#p age//10/06/19/100619803724793270162039958413564368089.jpg/mode/1up.

17. Nous restons favorable à une datation ancienne du Psautier d'Utrecht. Voir sur ce point J.-P. CAILLET, L'Art carolingien..., p. 180. 
L'image du Psautier d'Utrecht a en effet servi de modèle à l'ivoirier qui a sculpté la plaque d'ivoire qui orne le plat inférieur de la reliure du Psautier de Charles le Chauve ${ }^{18}$. Plus resserrée, elle est aussi plus nerveuse et probablement plus démonstrative. Au registre inférieur nous retrouvons la parabole de l'homme pauvre et de l'homme riche. Au registre médian, pour séparer les deux parties de la plaque, le cadavre d'Urie, admirablement modelé. Au registre supérieur, Bethsabée, David et Nathan. Plus nettement que dans le prototype, la colère prophétique est rendue visible par le bouillonnement des plis du manteau, le geste de la main droite du prophète et l'expression de son visage. Mais il y a plus intéressant pour notre propos. Dans le second cas, David a perdu sa couronne. Or, la couronne est probablement de tous les insignes royaux celui qui est le plus lié à la relation entre Dieu et le monarque et à l'accomplissement correct, selon le droit (comme l'écrit Isidore de Séville), du ministère royal ${ }^{19}$. Donc un adultère doublé d'un abus de pouvoir peut priver le prince de son ministère.

Le peintre du Psautier de Stuttgart a lui aussi illustré le psaume $50^{20}$. À la différence de son homologue rémois, au lieu de concentrer tout le psaume en une seule image, complexe le plus souvent, il choisit de représenter chacun des versets auxquels il entend donner une illustration par une enluminure autonome. C'est ici le cas et il est frappant de remarquer que les seules figures qui pourraient évoquer l'épisode que nous venons d'analyser sont celles que l'on retrouve sous le verset 8 du psaume qui traite de la conception peccamineuse du psalmiste. L'image n'est pas particulièrement explicite et il n'est pas évident de distinguer David de Bethsabée. Si le personnage drapé du manteau orange pourrait être la mère de Salomon, celui dans le manteau pourpre pourrait être David, David psalmiste et pénitent s'interrogeant sur sa faute tandis que Nathan, pieds nus comme il sied à un prophète, se dirige vers le temple. Mais les chaussures ouvragées des deux personnages rendent difficile la séparation de sexes, à moins d'oser une hypothèse particulièrement hasardeuse et de chercher la solution du problème dans l'enluminure suivante ${ }^{21}$. Cette dernière illustre le psaume 51 , dont

18. Psautier de Charles le Chauve, Paris, BnF, ms. lat. 1152, plat inférieur de la reliure. http:// visualiseur.bnf.fr/Visualiseur?Destination=Mandragore $\& \mathrm{O}=08003586 \& \mathrm{E}=1 \& \mathrm{I}=120641 \& \mathrm{M}=\mathrm{imag}$ eseule. Pour la datation: Trésors carolingiens, Paris, 2007, $\mathrm{n}^{\circ}$ 15, p. 108-112.

19. IsIDORE DE SÉviLLE, Etymologiae, éd. W. M. LindSAY, Oxford, 1911, p. 25 : «ut reges a recte agendo».

20. Psautier de Stuttgart, $\mathrm{f}^{\circ} 63 \mathrm{v}^{\circ}$, http://digital.wlb-stuttgart.de/digitale-sammlungen/ seitenansicht $/ ? \mathrm{id}=4870 \& \mathrm{tx} \_\mathrm{dlf} \% 5 \mathrm{Bid} \% 5 \mathrm{D}=1517 \& \mathrm{tx} \_\mathrm{dlf} \% 5 \mathrm{Bpointer} \% 5 \mathrm{D}=0 \& \mathrm{tx} \_\mathrm{dlf} \% 5 \mathrm{Bpage} \%$ $5 \mathrm{D}=130$.

21. Ibid., $\mathrm{f}^{\mathrm{0}} 64 \mathrm{v}^{\mathrm{o}}, \mathrm{http} / / /$ digital.wlb-stuttgart.de/digitale-sammlungen/seitenansicht/?id=4870\& tx_dlf\%5Bid\%5D=1517\&tx_dlf\%5Bpointer\%5D=0\&tx_dlf\%5Bpage $\% 5 \mathrm{D}=132$. 
le verset 2 rappelle la dénonciation de David par Doëg l’Édomite ${ }^{22}$. Le psaume porte d'ailleurs, dans les éditions modernes de la Vulgate, le titre «Contre le faux accusateur très puissant ${ }^{23}{ }$. Si le prince, assis sur un trône en X, écoute le calomniateur, derrière eux se déroule une scène qui n'a aucun rapport avec le texte du psaume 51 . Un homme nu et sexué ${ }^{24}$ frotte le corps d'une femme. Derrière eux un récipient contient du liquide. Ce pourrait être une synthèse iconographique du verset 2 du psaume 51 et du verset 9 du psaume 50 qui dit «ôte mon péché avec l'hysope, je serai pur; lave-moi, et je serai plus blanc que neige ${ }^{25} »$. On pourrait alors se demander, mais nous reconnaissons encore le caractère hypothétique de cette proposition, si le repentir du pécheur du folio 63 verso ne se retrouve pas dans la purification corporelle du folio 64 verso.

La signification de l'adultère, telle qu'elle apparaît dans ces documents, ne prendra réellement son sens que dans un dernier temps. Avant d'y parvenir, il faut analyser quelques images de couples légitimes, et notamment quelques représentations carolingiennes du mariage. La documentation viendra, bien entendu, des deux psautiers que nous étudions principalement.

\section{Des couples sans prêtre}

Pour des questions de cohérence, nous allons suivre l'ordre des psaumes, en passant de l'un à l'autre manuscrit. La première image qui nous retiendra est celle choisie pour illustrer le verset 10 du psaume 26. Il s'agit du folio 33 verso du Psautier de Stuttgart ${ }^{26}$. Le psalmiste qui dit vouloir «habiter la maison de Yahvé ${ }^{27}$ » souligne que ce dernier l'accueille alors que: «[son] père et [sa] mère l'abandonnent ${ }^{28} \gg$. Le couple est ici représenté; le geste du père, qui regarde la mère, désigne le fils. À l'inverse, la femme détourne les yeux de la scène. Le psalmiste se retourne pour regarder ses parents. Le couple est donc ici réduit à la parentalité, ce qui n'est guère étonnant. L'image proposée dans le Psautier d'Utrecht ${ }^{29}$ est en revanche plus intéressante, car il s'agit là d'une des

22. Ps. 51, v. 2: «Cum venit Doeg Idumaeus et nunutiavit Sauli: Venit David in domum Achimelech» (Quand Doëg l'Édomite vint annoncer à Saül: «David est entré dans la maison d'Ahimélek»).

23. «Contra calumniatorem praepotentem».

24. Les parties génitales ont été grattées ici comme ailleurs dans le manuscrit.

25. Ps. 50, v. 9: «Asperges me hyssopo, et mundabor; lavabis me, et super nivem dealbabor».

26. http://digital.wlb-stuttgart.de/digitale-sammlungen/seitenansicht/ $\mathrm{id}=4870 \& \mathrm{tx}$ dlf\%5Bid\%5D=1517\&tx_dlf\%5Bpointer\%5D=0\&tx_dlf\%5Bpage\%5D=70.

27. Ps. 26, v. $4:$ : Ut inhabitem in domo Domini omnibus diebus vitae meae».

28. Ps. 26, v. 10: «Quoniam pater meus et mater mea derelequerunt me».

29. Psautier d'Utrecht, $\mathrm{f}^{\mathrm{o}} 15 \mathrm{r}^{\mathrm{o}}$, http://objects.library.uu.nl/reader/index.php?obj=1874-99999 \&lan=en\#page//psalter/FOL_015R.jpg/mode/1up. 
rares images de vie familiale que nous conservons, à notre connaissance, dans l'iconographie carolingienne. Comme à son habitude, le dessinateur démultiplie les personnages: le psalmiste est accueilli au centre de l'image par le Christ au seuil de la «maison de Yahvé», mais il est aussi représenté enfant auprès de son père et de sa mère. Devant une maison à l'ombre d'un arbre, un couple se tient par la main et l'enfant se saisit de la main droite de sa mère. Si l'enfant regarde ses parents, ces derniers tournent les yeux vers la scène centrale.

L'image du folio 41 verso du Psautier de Stuttgart ${ }^{30}$ illustre le verset 12 du psaume 33: «Fils, venez m'écouter! Je vous enseignerai la crainte du SEIGNEUR ${ }^{31}$.» Un personnage nimbé est en situation d'enseignement, assis sur un trône bahut carré. Deux enfants prosternés baisent ses pieds. Derrière eux un homme et une femme. Visiblement, il s'agit une fois encore de leurs parents.

Le psaume 44 offre, bien évidemment, à celui qui traque des représentations de couples un morceau de choix. Il est vrai qu'il est qualifié d'Épithalame royal ${ }^{32}$. Le texte du psaume mériterait d'être l'objet d'une étude afin de dégager les enjeux politiques que l'époque carolingienne pouvait y enclore, mais ce n'est pas l'objet de cette recherche. Voyons tout d'abord l'enluminure que nous propose le Psautier de Stuttgart ${ }^{33}$. À gauche, tête nue, une figure emblématique, qui pourrait figurer l'abondance, tient une coupe et une palme. Il faut peut-être voir dans ce personnage une illustration des allusions faites par le verset 17 du psaume 44 aux fils du roi qui succéderont à leurs ancêtres ${ }^{34}$. Ce thème de l'abondance des fils est courant dans la littérature parénétique: que l'on veuille bien se souvenir du Pseudo-Cyprien ${ }^{35}$ ou de la lettre d'Alcuin de 793 adressée, entre autres, à Æthelbred et qui évoque «l'abondance de la terre, la prospérité des fils ${ }^{36} \gg$. Au centre de la composition, le couple. Le roi ${ }^{37}$ enlace la reine ${ }^{38}$. L'un et l'autre sont couronnés, l'un et l'autre de pourpre vêtus. Le prince tient

30. http://digital.wlb-stuttgart.de/digitale-sammlungen/seitenansicht/?id=4870\&tx_dlf\% 5Bid\%5D=1517\&tx_dlf\%5Bpointer\%5D=0\&tx_dlf\%5Bpage\%5D=86.

31. Ps. 33, v. 12: «Venite, filii, audite me; timorem Domini docebo vos.»

32. Ps. 44: «Carmen nuptiale regis Messiae.»

33. Psautier de Stuttgart, $\mathrm{f}^{\circ} 57 \mathrm{v}^{\circ}$, http://digital.wlb-stuttgart.de/digitale-sammlungen/ seitenansicht $/ ? \mathrm{id}=4870 \& \mathrm{tx} \_\mathrm{dlf} \% 5 \mathrm{Bid} \% 5 \mathrm{D}=1517 \& \mathrm{tx} \_\mathrm{dlf} \% 5 \mathrm{~B}$ pointer $\% 5 \mathrm{D}=0 \& \mathrm{tx} \_\mathrm{dlf} \%$ 5Bpage $\% 5 \mathrm{D}=118$.

34. Ps. 44, v. 17: «Pro patribus tuis nati sunt tibi filii; Constitues eos principes super omnem terram» (Tes fils remplaceront tes pères, tu en feras des princes sur toute la terre).

35. Pseudo-Cyprien, De XII abusivis saeculi (éd. S. Hellmann, Leipzig, 1909, p. 52-53). Le texte est trop long pour être intégralement cité.

36. Alcuin, Epistolae, ep. 18 (éd. E. DümmLer, Berlin, 1896, p. 51) : «Legimus quoque, quod regis bonitas totius est $[\ldots]$ terrae habundantia, filiorum benedictio».

37. Ps. 44, v. 12: «Et concupiscet rex decorem tuum» (que le roi s'éprenne de ta beauté).

38. Ps. 44, v. 10 : «Filiae regum in honore tuo. Astitit regina a dextris tuis» (Des filles de rois sont là... debout, à ta droite, la dame...). 
de surcroît un sceptre. L'ange qui ferme la composition tient aussi un sceptre proche typologiquement de celui du monarque. Une autre image fait face à celle que nous avons examinée et complète l'illustration du psaume $44^{39}$. On y voit la reine précédée d'un garde. Suivie d'une des «regi virgines ${ }^{40} »$, elle s'avance vers le palais royal. Venons-en maintenant à la lecture qu'a faite du même psaume l'artiste qui a réalisé le Psautier d'Utrecht ${ }^{41}$.

L'analyse portera sur la scène principale, celle qui se déroule devant le palais royal. Cette fois, le prince n'est plus muni du sceptre mais d'une épée, comme le lui enjoint le verset $4^{42}$. Un trône est au centre du palais entre les deux ailes. Devant celle de gauche se trouve la reine, vêtue d'une somptueuse robe qui fait penser à celle de la femme d'Harold dans le récit qu'Ermold le Noir donne du baptême des Danois ${ }^{43}$. Si nous comparons les deux images, il y a une disparition, l'ange, et un ajout: la corne que tient la main de Dieu ${ }^{44}$. Un jet d'huile en coule et vient oindre le front du roi couronné. Nous reviendrons plus loin sur la signification de ce geste. Soulignons l'absence de tout membre du clergé, alors que le texte du psaume aurait pu requérir leur présence.

Le folio 73 verso du Psautier d'Utrecht offre une illustration tout à fait remarquable du psaume 127 qui énonce les bienfaits promis à ceux qui craignent le Seigneur ${ }^{45}$. Se fondant sur les versets 2 et 3 - «Ta femme est une vigne généreuse au fond de ta maison, tes fils, des plants d'olivier autour de la table ${ }^{46} \gg-$, l'illustrateur a dessiné une scène saisissante du bonheur conjugal carolingien. Un couple royal, alors que rien dans le texte ${ }^{47}$ ne l'appelait, est

39. Psautier de Stuttgart, fo $58 \mathrm{r}^{\circ}$, http://digital.wlb-stuttgart.de/digitale-sammlungen/

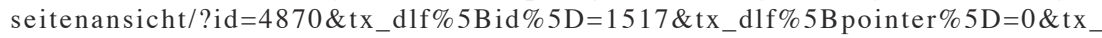
dlf\%5Bpage $\% 5 \mathrm{D}=119$.

40. Ps. 44, v. 15: «Adducentur regi virgines post eam» (les demoiselles... sont introduites auprès du roi).

41. Psautier d'Utrecht, Utrecht, Rijksuniversiteit te Utrecht. Bibliotheek, ms.32, fo $26 \mathrm{r}^{\circ}$. http://objects.library.uu.nl/reader/index.php?obj=1874-284427\&lan=en\#p age//16/70/40/167040758242302384976208542573921469544.jpg/mode/1up.

42. Ps. 44, v. 4 : «Accingere gladio tu super femur tuum» (ceins ton épée au côté).

43. ERmold le Noir, In honorem Hludovici, v. 2266-2269 (éd. et trad. E. FARAL, Poème en l'honneur de Louis le Pieux, Paris, 1932, p. 172-175): «Munera praeterea matronae regia Judith/ Congrua namque dedit gratificum decus,/Scilicet ex auro tunicam gemmisque rigentem/Conficit ast qualem arte Minerva sua» (De la reine Judith l'épouse du prince reçoit les présents magnifiques qui conviennent à son rang: elle revêt une tunique chargée d'or et de pierres précieuses, comme Minerve en eût pu tisser une).

44. Ps. 44, v. 8 : «Propterea unxit te Deus» (Aussi Dieu t'a oint...).

45. http://objects.library.uu.nl/reader/index.php?obj=1874-99999\&lan=en\#page//psalter/ FOL_073V.jpg/mode/1up.

46. Ps. 127, v. 2-3: «Uxor tua sicut vitis abundans, in lateribus domus tuae./Filii tui, sicut novellae olivarum in circuitu mensae tuae».

47. Qui n'est pas de David. 
entouré de ses fils. Cette image peut nous conduire vers notre troisième partie, où il sera question du couple royal et de sa descendance.

\section{Des enfants pas comme les autres}

Si nous regardons le frontispice de la Bible de Saint-Paul-hors-les-Murs ${ }^{48}$, réalisée alors que Charles le Chauve peut raisonnablement nourrir des ambitions impériales ${ }^{49}$, nous y voyons une femme, située à la gauche du monarque et qui fait deux gestes particulièrement signifiants.

Avant de les détailler, il convient d'identifier les protagonistes. Le travail est grandement facilité par le globe, tenu par le prince dans sa main gauche, qui porte l'inscription «Christe, conserve Karolum et Richildim». Nous sommes donc en présence de Charles le Chauve et de sa seconde épouse Richilde ${ }^{50}$. L'enceinte qui entoure le monarque le sacralise en le séparant de son entourage. Sacralisation qui rappelle sa fonction de roi médiateur ${ }^{51}$, mais aussi sa mission de roi eschatologique ${ }^{52}$. La main droite de Richilde est au contact de cet édifice tandis que sa gauche vient toucher son ventre. Or, ce dernier est nettement celui d'une femme qui attend un enfant. Le doute, s'il en subsistait un, est levé dès lors que l'on lit le poème situé sous l'image, qui évoque la proles ${ }^{53}$, la lignée, les enfants qui seront mis au monde, rite, selon le rite, par la reine ${ }^{54}$.

En décrivant Charles le Chauve trônant, nous avons rappelé sa mission eschatologique. Or, cette dernière est liée à la possibilité, offerte au prince par le sacre royal, d'engendrer des rois qui constitueront une nouvelle dynastie de

48. Bible de Saint-Paul-hors-les-Murs. Rome, Saint-Paul-hors-les-Murs, $\mathrm{f}^{\circ} 1 \mathrm{r}^{\circ}$.

49. Voir sur ce point D. Alibert, Les Carolingiens et leurs images..., p. 238-239.

50. C'est Percy Ernst Schramm qui a proposé cette lecture. Il avait déchiffré Richardim pour le prénom féminin mais admettait fort bien la lecture Richildim. C'est cette dernière que proposait Ernst Hartwig Kantorowicz. P. E. Schramm, «Umstrittene Kaiserbilder aus dem 9-12 Jahrhundert», Neues Archiv der Gesellschaft für ältere deutsche Geschichtskunde, 74 (1928), p. 479; E.-H. Kantorowicz, «The Carolingian King in the Bible of San Paolo fuori le mura», Late Classical and Medieval Studies in Memory of Albert Mathias Friend Jr, Princeton, 1955, p. 290.

51. D. Alibert, Les Carolingiens et leurs images..., p. 477-481.

52. Sur cette dernière, une première introduction: D. AlibERT, «Naissance des idéologies médiévales dans les images politiques carolingiennes», dans E. SAntinelli-Foltz, C.-G. Schwentzel éd., La Puissance royale. Image et pouvoir de l'Antiquité au Moyen Âge, Rennes, 2011, p. 85-97.

53. Outre les remarques que nous faisons sur ce terme dans les différents articles cités ci-après, voir E. Deноux, «Une simple affaire de famille? Usage et portée du mot "proles" dans la France de l'Ouest (IXe-XII" s.)», Bulletin de la Société archéologique du Finistère, 140 (2012), p. 241-266.

54. Tituli de la Bible de Saint-Paul-hors-les-Murs: «Nobilis ad levam coniux de more venustat/ Qua insignis proles in regnum rite paretur». Tituli de la Bible de Saint-Paul-hors-les-Murs, éd. L. Traube, Berlin, Weidmann, 1896, p. 257. 
Juda et régneront jusqu'au retour du roi messie ${ }^{55}$. Cette mission donne tout leur sens à deux documents. Nous avons déjà évoqué l'un d'eux, le folio 26 recto du Psautier d'Utrecht ${ }^{56}$. Nous avions incidemment fait remarquer que, pour illustrer le psaume 44, l'artiste qui avait œuvré à Reims s'était distingué de celui qui avait enluminé le Psautier de Stuttgart en n'omettant pas la scène de l'onction appelée par le verset 8 . En revanche, nous n'avons encore rien dit du folio $51 v^{\circ}$ du Psautier d'Utrecht qui illustre le psaume $88^{57}$. La proximité des textes, mais aussi une certaine parenté des images, peuvent inciter à un rapprochement qui, s'il peut paraître audacieux, pourrait expliquer le fait que ce sont essentiellement les couples royaux qui sont représentés dans l'iconographie carolingienne.

Quels sont les éléments qui permettent d'établir un lien entre ces deux passages ? Prenons le psaume 44, verset 7 - «Ô Dieu, ton trône est éternel ${ }^{58}$ » - et le psaume 88 versets 4-5 et 37 - «j'ai conclu une alliance avec mon élu, j'ai juré à David mon serviteur:/j'établis ta dynastie pour toujours, je t'ai édifié un trône pour tous les siècles ${ }^{59} »$; «Sa dynastie durera toujours et son trône sera devant moi, comme le soleil ${ }^{60} »$. La similitude entre les deux textes est, nous semble$\mathrm{t}$-il, assez nette pour tenter un rapprochement entre le psaume sur le mariage et celui sur l'alliance entre Dieu et son élu. Mais il y a plus. Le psaume 44 et le psaume 88 sont les deux seuls dans tout le psautier à utiliser le verbe «oindre». Dans le psaume 44, au verset 8, comme nous l'avons vu, Dieu dit au roi messie: «Dieu, ton Dieu, t'a oint ${ }^{61}$ », tandis que le verset $21 \mathrm{du}$ psaume 88 dit: «j'ai trouvé David mon serviteur, je l'ai sacré avec mon huile sainte ${ }^{62} »$. L'un et l'autre psaume évoquent, comme cela a été signalé, la descendance du prince, la pérennité de sa dynastie.

Si l'on replace ces deux images ${ }^{63}$, qui présentent une certaine similitude (avec notamment le décor et l'apparat de la fonction royale), dans le contexte

55. Sur ce point, D. AliBERT, «Semen eius in aeternum manebit. Remarques sur l'engendrement royal à l'époque carolingienne», dans M. Rouche éd., Sexualité et mariage au Moyen Âge, accord ou crise?, Paris, 2000, p. 137-147.

56. Psautier d'Utrecht, Utrecht, Rijksuniversiteit te Utrecht. Bibliotheek, ms. 32, fo $26 \mathrm{r}^{\circ}$. http://objects.library.uu.nl/reader/index.php?obj=1874-284427\&lan=en\#p age//16/70/40/167040758242302384976208542573921469544.jpg/mode/1up.

57. Ibid. $\mathrm{f}^{\circ} 51 \mathrm{r}^{\mathrm{o}}$. http://objects.library.uu.nl/reader/index.php?obj=1874-284427\&lan=en\#p age//16/46/54/164654509020680716632913951820563636455.jpg/mode/1up.

58. Ps. 44, v. 7 : «sedes tua, Deus, in saeculum saeculi».

59. Ps. 88 , v. 4-5: «Disposui testamentum electis meis; juravi David, servo meo/usque in aeternum praeparabo semen tuum,/Et aedificabo in generationem et generationem sedem tuam ».

60. Ps. 88, v. 37: «semen eius in aeternum manebit et thronus eius sicut sol in conspectu meo».

61. Ps. 44, v. 8 : «unxit te Deus, Deus tuus».

62. Ps. 88 , v. 21 : «Inveni David, servum meum oleo sancto meo unxi eum».

63. Psautier d'Utrecht, Utrecht, Rijksuniversiteit te Utrecht. Bibliotheek, ms. 32 , fo $26 \mathrm{r}^{\circ}$ (http://objects.library.uu.nl/reader/index.php?obj=1874-284427\&lan=en 
idéologique carolingien, si on les rapproche du message de la Bible de Saint-Paulhors-les-Murs, dont on a pu montrer une certaine parenté avec des manuscrits rémois, l'ensemble pourrait offrir une certaine cohérence. Le couple royal carolingien, héritier de la promesse faite à David dans le psaume 88, marié afin que lui viennent des fils qui seront des princes par toute la terre, comme le promet le psaume 44, peut engendrer cette proles, cette descendance qui devra conserver le sceptre jusqu'à ce que vienne celui à qui il appartient, le Christ des fins dernières. Il est un autre indice qui permettrait d'aller dans cette direction. Si à Reims, David, nu, est oint par des anges ${ }^{64}$, à Saint-Germain-des-Prés, où il est tout aussi nu, c'est un prophète qui procède à l'onction ${ }^{65}$. Dans le premier cas ce sont des anges qui tiennent les vêtements du roi, tandis que dans le second c'est une femme. Or la robe qu'elle porte est bleu pâle, tandis que le vêtement royal est d'un bleu plus saturé. De là à penser qu'il s'agit de la reine, il n'y a qu'un pas que l'absence de couronne empêche de franchir, mais l'hypothèse, tentante, doit être émise.

Une fois encore, le système idéologique carolingien nous montre sa complexité. Car les couples que nous avons étudiés représentent-ils ce couple royal que forment tous les couples de baptisés, puisque, comme l'écrit l'apôtre Pierre, tous les baptisés constituent un peuple de rois, un sacerdoce royal ${ }^{66}$ ? Ou au contraire, faut-il voir dans ces images le rappel de la mission eschatologique propre, depuis l'invention du sacre, à la dynastie carolingienne ${ }^{67}$ ? La puissance et l'efficacité de ce système se résument à cette seule question.

Mais il y a plus. Si le lien que l'on a tenté d'établir entre mariage et sacre royal est plausible ${ }^{68}$, cela expliquerait pourquoi les moralistes carolingiens combattent avec tant d'insistance l'adultère, au point de rappeler que David luimême céda à la tentation. À moins qu'il ne s'agisse pour eux de souligner que le prince pécheur n'est qu'un pénitent comme tant d'autres, ce qui expliquerait

\#page//16/70/40/167040758242302384976208542573921469544.jpg/mode/1up) et $\mathrm{f}^{\circ} 51 \mathrm{v}^{\circ}$ (http://objects.library.uu.nl/reader/index.php?obj=1874-284427\&lan=en\#p age//16/46/54/164654509020680716632913951820563636455.jpg/mode/1up).

64. Ibid., f $\mathrm{f}^{\circ} 51 \mathrm{v}^{\circ}$.

65. Psautier de Stuttgart, $\mathrm{f}^{\circ} 104 \mathrm{r}^{\mathrm{o}}$, http://digital.wlb-stuttgart.de/digitale-sammlungen/ seitenansicht $/$ ? id $=4870 \&$ tx_dlf $\% 5 \mathrm{Bid} \% 5 \mathrm{D}=1517 \& \mathrm{x} \_\mathrm{dlf} \% 5 \mathrm{Bpointer} \% \mathrm{D}=0 \& \mathrm{tx} \_\mathrm{dlf} \% 5 \mathrm{Bpage} \%$ $5 \mathrm{D}=213$.

66. Première Épître de Pierre, 2, 9: «vos autem genus electum, regale sacerdotium, gens sancta» (vous, vous êtes la race élue, la communauté sacerdotale du roi, la nation sainte).

67. D. AliberT, «Procéder au sacre royal à l'époque carolingienne», dans J. Hoareau-Dodinau, G. Métairie et P. TeXIer éd., Procéder. Pas d'action, pas de droit ou pas de droit, pas d'action?, Limoges, 2006, p. 85-95.

68. D. AliberT, «Sacre royal et onction royale à l'époque carolingienne», dans J. HoAREAUDodinau et P. Texier éd., Anthropologies juridiques. Mélanges Pierre Braun, Limoges, 1998, p. 28-32. 
selon nous, mais ce point mériterait d'amples développements, la présence de la remontrance de Nathan sur la reliure du Psautier de Charles le Chauve.

Ces questions prouvent en tout cas que ceux qui ont conçu ce système savaient transformer le mariage en une alliance éternelle. Ils n'ont pas fini de susciter les travaux des historiens.

Dominique AliberT - Faculté des Lettres, Institut Catholique de Paris, 21 rue d'Assas, 75270 Paris Cedex 06

\section{Un homme, une femme et des enfants :}

\section{autour du couple dans quelques images du haut Moyen Âge}

À partir d'un corpus iconographique relativement réduit présentant des couples extraordinaires, David et Bethsabée, Charles le Chauve et Richilde, mais aussi des époux ordinaires, l'étude s'attache à définir la place que l'iconographie carolingienne entend attribuer au couple. Si la fonction parentale et la condamnation de l'adultère sont mises en avant, ce n'est pas exclusivement pour des questions morales mais aussi parce que la dynastie carolingienne a la mission de conduire le peuple franc, nouveau peuple élu, vers ses destinées finales.

royauté - eschatologie - sacre royal - IX $\mathrm{X}^{\mathrm{e}}$ siècle - famille

\section{Man, Woman and Children :}

\section{the Couple in a Few Images of the Early Middle Ages}

From a relatively reduced iconographic corpus presenting extraordinary couples, David and Bethsabee, Charles the Bald and Richilde, but also ordinary spouses, this study attempts to define the place which the Carolingian iconography intends to attribute to the couple. If the parental function and the condemnation of the adultery are put forwards, it's not exclusively for moral questions, but also because the Carolingian dynasty has the mission to lead the Franks, new elected people, towards its final destiny.

kingship - eschatology - royal coronation - ninth century - family 
